\title{
Role of trapping and crowding as sources of negative differential mobility
}

\author{
Marco Baiesi, ${ }^{1,2, *}$ Attilio L. Stella, ${ }^{1,2}$ and Carlo Vanderzande ${ }^{3,4}$ \\ ${ }^{1}$ Department of Physics and Astronomy, University of Padova, Via Marzolo 8, I-35131 Padova, Italy \\ ${ }^{2}$ INFN, Sezione di Padova, Via Marzolo 8, I-35131 Padova, Italy \\ ${ }^{3}$ Faculteit Wetenschappen, Hasselt University, 3590 Diepenbeek, Belgium \\ ${ }^{4}$ Instituut theoretische fysica, Katholieke Universiteit Leuven, 3001 Leuven, Belgium
}

(Received 8 September 2015; published 9 October 2015)

\begin{abstract}
Increasing the crowding in an environment does not necessarily trigger negative differential mobility of strongly pushed particles. Moreover, the choice of the model, in particular the kind of microscopic jump rates, may be very relevant in determining the mobility. We support these points via simple examples and we therefore address recent claims saying that crowding in an environment is likely to promote negative differential mobility. Trapping of tagged particles enhanced by increasing the force remains the mechanism determining a drift velocity not monotonous in the driving force.
\end{abstract}

DOI: 10.1103/PhysRevE.92.042121

PACS number(s): 05.40.-a, 05.70.Ln

\section{INTRODUCTION}

In a system at thermodynamic equilibrium, a particle responds to a gentle push by moving, on average, toward the direction of the applied force. Therefore, the mean-velocityto-force ratio, called mobility, is a positive quantity. In general, linear response coefficients are positive in equilibrium because the structure of the fluctuation response relations involve positive correlations between observables and the entropy that perturbations would produce [1]. Far from equilibrium there is more variability. For example, thermal conductivities that are not monotonous in the external forcing have been found [2].

If a pushed particle starts to go slower by increasing the force, then we have the phenomenon of negative differential mobility (NDM) [3-12]. If the particle responds by drifting against the force, we speak of absolute negative mobility. The latter behavior has been observed in models subject to ratchet effects [13], in systems driven by periodic forces [14,15], and for driven Janus particles in corrugated channels [16].

Here we focus on NDM. Negative differential conductivity was discovered in the 1960s and realized in Gunn diodes [17] and other semiconductors at low temperature [17-19]. Other examples of NDM are listed in Ref. [10]. A recent application of NDM is the sorting of soft matter colloidal particles [20].

For common overdamped diffusion, NDM is expected when particles' surrounding environment contains shallow barriers, called "traps" hereafter, in which the particle driven by an external force might spend more and more time upon an increase of the force. For this reason, the mobility of the particle may actually decrease rather than increase if the force is raised. An example of this effect was given by particles diffusing within a percolating cluster [3] (an instance of hopping process in disordered media $[21,22]$ ), in which traps are represented by the dangling ends and all portions with concavity against the direction of the force. A pedagogical exemplification of this "getting more from pushing less" phenomenon was then given by Zia and coworkers [4]. In a two-lanes jump system that can be solved exactly, NDM emerges when one lane contains a hook that can trap the

*Corresponding author: baiesi@pd.infn.it particle. The NDM may be described [8,9,11] from the point of view of a recently developed linear response theory for general nonequilibrium systems [23-25]. In this context, NDM emerging in simulations of ring polymers impaled by rods of a lattice with defects [11] might explain irregular migration speeds in experiments comparing linearized and circular DNA plasmids [26,27]. NDM was also found for particles diffusing in a crowded environment $[7,9,10]$.

One expects to observe NDM if there is a form of trapping due to a specific coupling between the tracers (tagged particle, polymer, etc.) and their environment. Jamming of particles, such as in kinetically constrained models $[5,6]$, may also be seen as a form of mutual trapping. However, just a density of obstacles does not say anything straightforward about such propensity to meet traps if the features of the obstacles and of the tracers are not explicitly specified. Yet, using the formalism of mesoscopic jump systems, it was recently proposed [10] that NDM should be a generic feature for particles strongly pulled in crowded environments.

We believe that shifting the focus from the trapping mechanism to the role of crowding is not functional to a better understanding of NDM. To support our point, in this paper we show that some of the results presented in Ref. [10], and more generally the appearance of NDM, may depend crucially on the choice of microscopic jump rates. Moreover, we use some simple models to show that crowding may be uncorrelated with NDM or even anticorrelated. This occurs when too many tagged particles are introduced in the system, so traps are saturated.

\section{JUMP RATES AND A BASIC EXAMPLE}

As in previous studies, the results are exposed in the context of Markov jump processes. The states of the system are discrete and evolve with jumps taking place in continuous time. More specifically, we have in mind subsets of the square lattice where the particle may occupy free sites and jump to the first neighbors if the transitions are allowed. Forbidden transition may occur if the target of the jump is already occupied or if there is an idealized wall separating the two sites involved in the jump. We use a force $F$ parallel to the $x$ axis and periodic boundary conditions in this direction. Hence, we monitor the 
TABLE I. Jump rates of the particle on the square lattice with periodic boundary conditions. Here $X=2\left(e^{F / 2}+e^{-F / 2}\right)$ and $Z=e^{F / 2}+e^{-F / 2}+2$, so all rates $=1 / 4$ for $F=0$.

\begin{tabular}{lcll}
\hline \hline Direction & Rate & \multicolumn{1}{c}{ Model A } & \multicolumn{1}{c}{ Model B } \\
\hline Right & $k^{+}$ & $e^{F / 2} / X \sim\left(1-\epsilon^{2}\right) / 2$ & $e^{F / 2} / Z \sim 1-2 \epsilon$ \\
Left & $k^{-}$ & $e^{-F / 2} / X \sim \epsilon^{2} / 2$ & $e^{-F / 2} / Z \sim \epsilon^{2}$ \\
Up or down & $k^{0}$ & $1 / 4$ & $1 / Z \sim \epsilon$ \\
\hline \hline
\end{tabular}

average velocity $v \equiv\langle v\rangle_{F}$ in the $x$ direction. The differential mobility $\mu \equiv d v / d F$ becomes negative in the regions where $v$ decreases for increasing $F$.

For the sake of simplicity we use a unit temperature times Boltzmann constant combination, $k_{B} T=1$, and unit spacing between sites of the square lattice. This means that the principle of local detailed balance [28] is met if the jump rate $k\left(\mathcal{C} \rightarrow \mathcal{C}^{\prime}\right)$ is related to the jump rate $k\left(\mathcal{C}^{\prime} \rightarrow \mathcal{C}\right)$ of the reversed transition by

$$
\frac{k\left(\mathcal{C} \rightarrow \mathcal{C}^{\prime}\right)}{k\left(\mathcal{C}^{\prime} \rightarrow \mathcal{C}\right)}=\exp \left[U(\mathcal{C})-U\left(\mathcal{C}^{\prime}\right)\right],
$$

where $U(\mathcal{C})$ is the energy associated to the configuration $\mathcal{C}$. When a particle is subject to a constant force $F$ and the jump $\mathcal{C} \rightarrow \mathcal{C}^{\prime}$ is in the direction of the force, the energy difference becomes the work done by the force, $U(\mathcal{C})-U\left(\mathcal{C}^{\prime}\right)=F$. When the jump is orthogonal to $F$, simply $U(\mathcal{C})=U\left(\mathcal{C}^{\prime}\right)$. There are many (actually infinite) choices of the jump rates that satisfy the local detailed balance condition.

Normalizing jump rates of all four exit directions from a site with a common denominator is a popular choice (see, e.g., Refs. $[4,7,10])$, possibly because it is elegant and resembles a partition function normalization of Boltzmann weights. As a consequence, the rate of jumps directed transversely to a large force $F$ scale as $\sim \exp (-F)$. Another possibility would be to keep transversal rates independent of $F$ [9]. We call model $A$ the latter and model $B$ the former. The details of these rates are given in Table I, where we also specify the scaling for large $F$ as a function of $\epsilon=e^{-F / 2}$. We pick up just these two variants among infinite ones because they already represent two distinct classes of dynamical behavior.

In order to have a quick taste of the qualitatively dissimilar mobilities that model $\mathrm{A}$ and $\mathrm{B}$ may give, let us introduce a minimal two-lanes model of particle diffusing in a meandering channel. This model includes two kind of states: $\mathcal{C}_{1}$ states where the force pushes the particle against a barrier (colored boxes in Fig. 1) and $\mathcal{C}_{2}$ states where the force pulls the particle away from a barrier (white boxes). One easily finds the density of states $p$ and the mean velocity of particles in the steady state,

$$
\begin{aligned}
p\left(\mathcal{C}_{1}\right) & =k^{+}+k^{0}, \\
p\left(\mathcal{C}_{2}\right) & =k^{-}+k^{0}, \\
v & =k^{+} p\left(\mathcal{C}_{2}\right)-k^{-} p\left(\mathcal{C}_{1}\right) .
\end{aligned}
$$

For models $\mathrm{A}$ and $\mathrm{B}$ the mean velocities are

$$
v_{\mathrm{A}}=\frac{1}{8} \tanh \frac{F}{2}, \quad v_{\mathrm{B}}=\frac{e^{F / 2}-e^{-F / 2}}{\left(e^{F / 2}+e^{-F / 2}+2\right)^{2}},
$$

respectively. The first is clearly increasing with $F$ while $v_{\mathrm{B}}$ cannot be monotonous in $F$ as $v_{\mathrm{B}} \sim \epsilon$ for $F \rightarrow \infty$ (Fig. 1

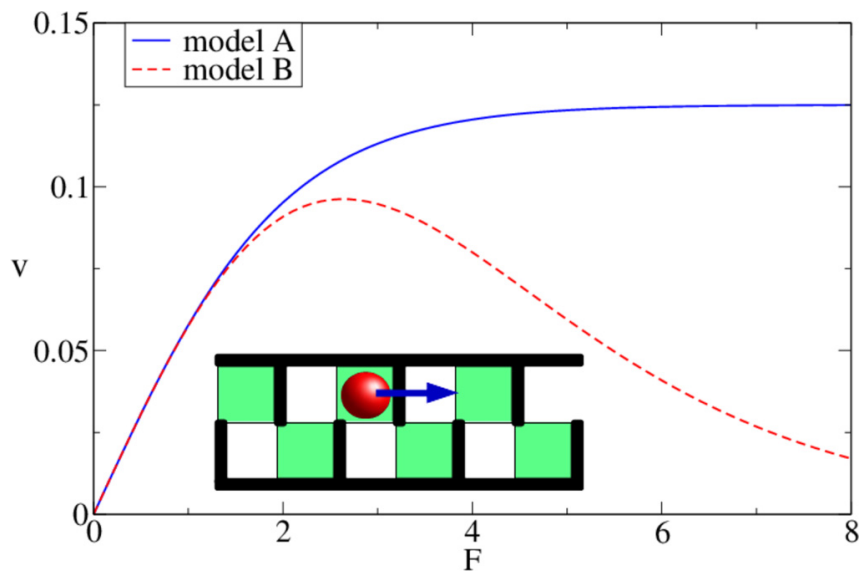

FIG. 1. (Color online) Sketch of the two-lanes toy model, and its mean velocity [see (3)] as a function of the force, for model A and model B.

shows the plots of both functions). Hence, model A does not yield NDM in this case, but model B does.

A similar fact pertains to scaling theories focused on the density of obstacles $[7,10]$. For example, the theory presented in Ref. [10] does not yield NDM if constant transversal rates are plugged in their Eq. (2) for the average velocity of the tracer, rather than those $\sim \epsilon$ of model B. Indeed, with model A rates, the same formula becomes a monotonously increasing function of $F$ and NDM disappears.

In the following section we show a similar effect for a variant of the two-dimensional diffusion within slowly moving barriers, which was recently used $[9,10]$ as a main benchmark for testing ideas on NDM.

\section{SINGLE TAGGED PARTICLE WITHIN FLOATING BARRIERS}

Model A was used [9] (with a slightly different normalization of rates) to show how NDM arises for particles moving in crowded environments when the (nonoverlapping) obstacles have low-enough mobility $\gamma$ : Given a configuration $\mathcal{C}$ with $N$ obstacles and 1 tagged particle [see an example in Fig. 2(a)], the bare escape rate from $\mathcal{C}$ is $\lambda(\mathcal{C})=\lambda_{1}+N \gamma$, where $\lambda_{1}=k^{+}+k^{-}+2 k^{0}$ is the escape rate of the particle from any site within an empty grid $\left(\lambda_{1}=1\right.$ for the rates summarized in Table I). We extract a waiting time $\tau$ according to an exponential distribution $p(\tau) \sim \exp [\lambda(\mathcal{C}) \tau]$. After the extracted $\tau$, a move is attempted, picking up the tagged particle with probability $1 / \lambda$ or a random barrier with probability $\gamma / \lambda$. The tagged particle is then moved with a random selection of the direction that follows the rates previously exposed, while a barrier tries to jump with probability $1 / 4$ to a random nearest neighbor. An update realizes the new configuration only if it is allowed, otherwise the configuration is unchanged and another waiting time is added to the already-spent $\tau$ until a valid new configuration is achieved. Such modified Gillespie algorithm [29] is very simple to implement and allows us to sample efficiently the dynamics of these models. Note that a time scale $\tau^{*}=1 / \gamma$ is associated to barriers' motion, as opposed to a time scale equal to 1 for the particle motion. 
(a)

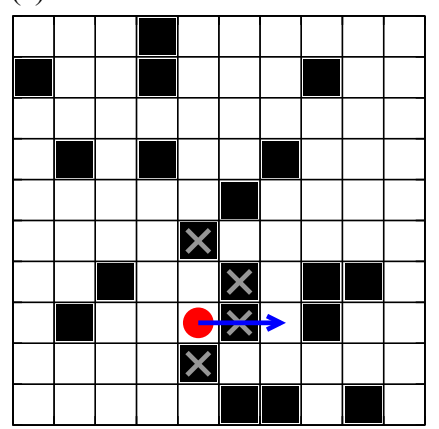

(b)

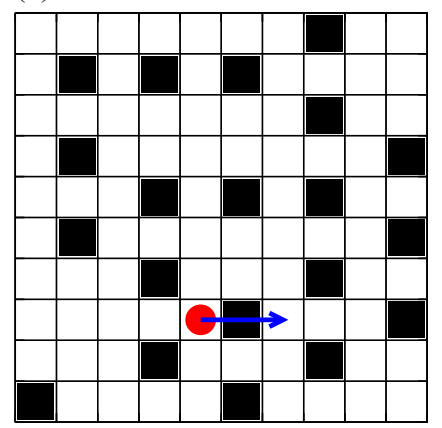

FIG. 2. (Color online) (a) Configuration with density of obstacles $\rho_{b}=0.2$. The circle (red online) is the tagged particle and the squares are the barriers. The four obstacles marked with a cross are forming a trap for the tracer. (b) Same $\rho_{b}=0.2$, when obstacles cannot have vertices overlapped.

We have performed simulations within square lattices of side $L$ filled with density $\rho_{b}=N / L^{2}$ of floating barriers. An example of a typical configuration for $\rho_{b}=0.2$ is shown in Fig. 2(a). In all simulation we find that both models A and B display a similar NDM at sufficiently large values of $F$.

However, it is not possible to switch with ease from one choice of microscopic jump rates to another. Indeed, even with the same crowding of obstacles, the situation changes drastically if these cannot share corners [Fig. 2(b)] and thus cannot join to form concave traps. In this case model A looses NDM while model B continues to display NDM (Fig. 3) because the tracer cannot easily move away from a situation as in Fig. 2(b), where the rates of model B lead to an effective trapping of the particle. By now this should be hardly surprising, as the constraint of nonoverlapping barrier's corners leads to morphologies analogous to those exemplified by the meandering channel model of the previous section, where no concavity is present and model A finds no traps to generate NDM. In the limit of low $\rho_{b}$ the corner constraint should be irrelevant. Yet theoretical approaches $[7,10]$ were

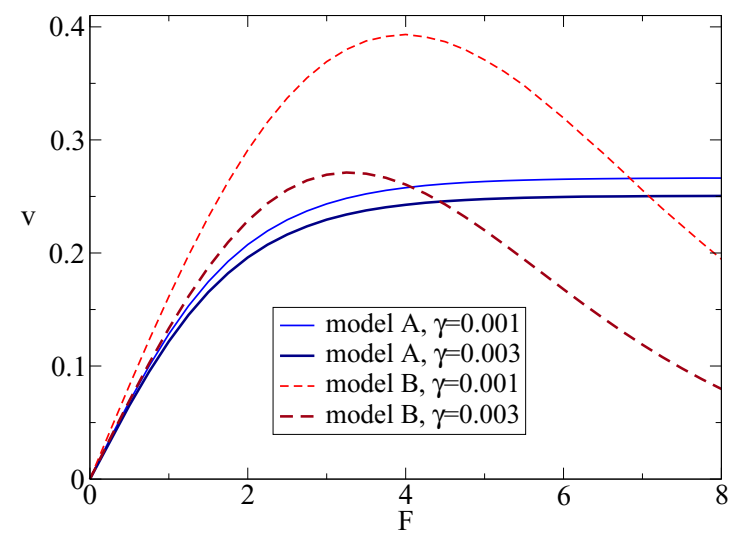

FIG. 3. (Color online) Mean velocity vs force for models A and B with nonoverlapping corners [case (b) of Fig. 2], for $L=20$, $\rho_{b}=0.2$, and two values of the obstacles' mobility $\gamma$. Similar results are found for other small $\gamma$ 's, in particular model A is quite insensitive to the value of $\gamma$. used to compute a nontrivial mobility also in the limit of low obstacle densities. Our results suggest that this procedure leads to an incomplete picture if only model B is considered.

\section{MULTIPLE TAGGED PARTICLES WITHIN FLOATING BARRIERS}

The crowding of an environment may result from the presence of different molecular species, and in general it is not straightforward to anticipate the mobility of particles in complex conditions. Here we may think of the supercrowded environment of the cell [30] which affects the diffusive properties of passive particles [31]. Much less is known about the behavior of active particles like molecular motors in such an environment [32].

As a first step toward more complex systems, in the simple model described in the previous section we may think the crowding not only determined by its barriers but also by a finite, possibly large, number of tracers. The total density of occupied sites in the system is thus the sum of the density of barriers $\rho_{b}$ and the density of tracer particles $\rho_{p}$. The resulting model is, more than that of Ref. [10], a combination of the symmetric and asymmetric exclusion processes. The condition of a finite yet low density of tracers seems similar to the condition of (a)

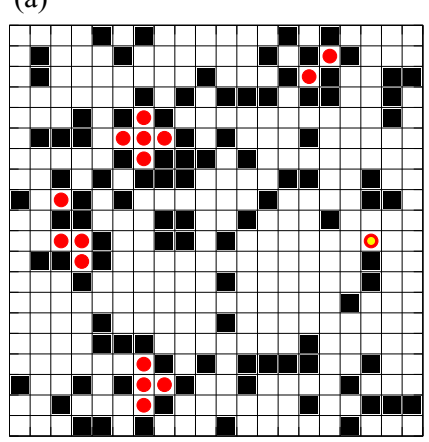

(b)

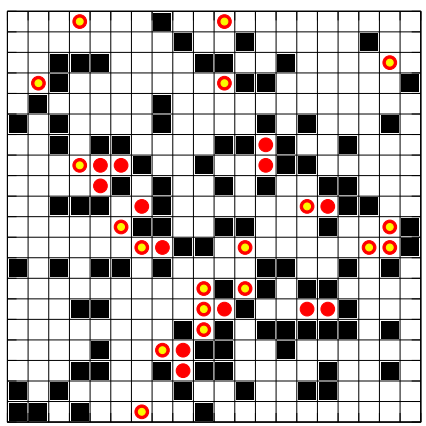

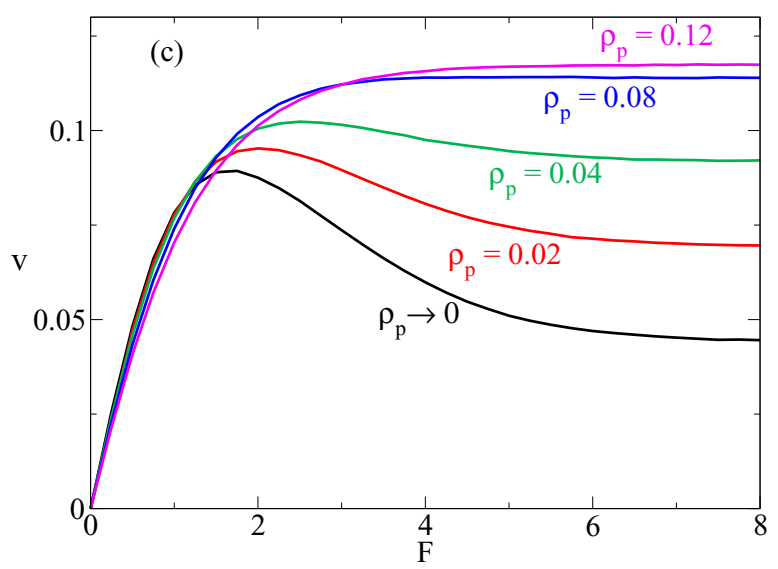

FIG. 4. (Color online) (a) Configuration for model A with $L=20, \rho_{p}=0.04, \rho_{b}=0.25, \gamma=10^{-3}$, and $F=8$ pushing particles to the right. Tracers that may follow $F$ by eventually never stepping back against it are drawn as empty circles (only one in this case). (b) The same for $\rho_{p}=0.08$. (c) Mean velocity vs force (same parameters) for several particle densities. 
low filling of bands of electrons, which leads to NDM in low-temperature semiconductors [19,21].

For model $\mathrm{A}$ it is not difficult to find conditions of low $\rho=\rho_{p}+\rho_{b}$ where NDM is present as well as conditions with high $\rho$ where NDM is not observed, see the examples in Fig. 4. The latter state of normal mobility arises because the behavior of tracers emerges as an average of two typical conditions, one of tracers stuck in traps and one of tracers free to move because all available traps are occupied by other tracers. For several values of $\rho_{b}$ we find that the contribution from running tracers determines a normal mobility for sufficiently high values of $\rho_{p}$. This is expected to occur when there are more particles than the average number of traps [e.g., as in Fig. 4(b)]. If instead $\rho_{p}$ is low enough, trapping is still the dominating behavior [see Fig. 4(a)].

This model therefore is an example where increased crowding leads to the disappearance of NDM. Again, this strengthens our point that the link between crowding and the mobility of particles is not straightforward. On the other hand, the numerical results may be correctly interpreted at least qualitatively in terms of saturation of trapping effects. Also in this case one could devise simpler systems where the mechanism of filling of all the traps is more easily detected. For example, in the two-lanes models where one lane is occupied by "hook" states that act as traps [4,8], we have numerically verified that NDM is present only if the number of tagged particles is at most equal to the number of traps.

\section{DISCUSSION AND CONCLUSIONS}

We have presented results from some simple models suggesting that the phenomenon of NDM in many cases can be ascribed to the presence of long-living traps that catch the strongly pushed traced particles. There is instead no one-to-one correspondence between crowding and NDM. In particular, according to our results, it seems far fetched to draw conclusions from either simulations or from mesoscopic theories if these rely on the specific choice of the jump rates.
The choice of transversal jump rates normalized so they decrease with the force is quite popular but it needs to be understood and justified. For example, starting from the concept of Brownian motion of a particle in a fluid, we do not see how the increasing force could lead to a weaker transversal motion, namely to a diffusion coefficient in the fluid that depends on a transversal $F$. Also for particles diffusing by jumping in a pattern of energy minima it is not general to find a decrease of transversal motion generated by an applied force. Think, for example, of a particle density within a minimum, at the microscopic level of description (while the mesoscopic level is the one of jump systems describing the energy minimum as a single state). If the applied force shifts the maximum of this density closer to the energetic saddle points that connect to nearby states, also in transverse directions, then we would witness a case where the force would actually enhance the transversal motion. It seems that such a scenario is in principle as likely as that where the transversal motion is depressed by the force. Hence, transversal jump rates that decrease exponentially with the applied force should be tested in parallel with other choices, keeping in mind that any specific system might be a realization of one of these choices.

Our examples also show that focusing on the density of objects responsible for the crowding is not a stand-alone strategy. One needs first to determine whether the tagged mobile objects (e.g., particles or polymers) might become more stuck by the crowded environment when the pushing force is increased. Furthermore, it is also relevant to check if, on average, the traps are at least as many as the tagged particles. The study of NDM in more complex simulations than those considered so far (such as the condition outlined in the previous section where multiple tracers coexists) should help to further clarify these issues. Of course, this is just part of a broader scenario where kinetic constraints, jamming, glassy dynamics, alternating external forces, self-propulsion, etc., furnish many more mechanisms leading to negative mobility.
[1] R. Kubo, M. Toda, and N. Hashitsume, Statistical Physics: Nonequilibrium Statistical Mechanics, 2nd ed. (Springer, Berlin, 1992), Vol. 2.

[2] B. Li, L. Wang, and G. Casati, Negative differential thermal resistance and thermal transistor, Appl. Phys. Lett. 88, 143501 (2006).

[3] D. Dhar, Diffusion and drift on percolation networks in an external field, J. Phys. A 17, L257 (1984).

[4] R. K. P. Zia, E. L. Praestgaard, and O. G. Mouritsen, Getting more from pushing less: Negative specific heat and conductivity in nonequilibrium steady states, Am. J. Phys. 70, 384 (2002).

[5] R. L. Jack, D. Kelsey, J. P. Garrahan, and D. Chandler, Negative differential mobility of weakly driven particles in models of glass formers, Phys. Rev. E 78, 011506 (2008).

[6] M. Sellitto, Asymmetric Exclusion Processes with Constrained Dynamics, Phys. Rev. Lett. 101, 048301 (2008).

[7] S. Leitmann and T. Franosch, Nonlinear Response in the Driven Lattice Lorentz Gas, Phys. Rev. Lett. 111, 190603 (2013).
[8] P. Baerts, U. Basu, C. Maes, and S. Safaverdi, Frenetic origin of negative differential response, Phys. Rev. E 88, 052109 (2013).

[9] U. Basu and C. Maes, Mobility transition in a dynamic environment, J. Phys. A 47, 255003 (2014).

[10] O. Bénichou, P. Illien, G. Oshanin, A. Sarracino, and R. Voituriez, Microscopic Theory for Negative Differential Mobility in Crowded Environments, Phys. Rev. Lett. 113, 268002 (2014).

[11] D. Michieletto, M. Baiesi, E. Orlandini, and M. S. Turner, Rings in random environments: Sensing disorder through topology, Soft Matter 11, 1100 (2015)

[12] G. Oshanin, J. Klafter, and M. Urbakh, Saltatory drift in a randomly driven two-wave potential, J. Phys.: Condens. Matter 17, S3697 (2005).

[13] R. Eichhorn, P. Reimann, and P. Hänggi, Brownian Motion Exhibiting Absolute Negative Mobility, Phys. Rev. Lett. 88, 190601 (2002).

[14] L. Machura, M. Kostur, P. Talkner, J. Łuczka, and P. Hänggi, Absolute Negative Mobility Induced by Thermal Equilibrium Fluctuations, Phys. Rev. Lett. 98, 040601 (2007). 
[15] F. Marchesoni and S. Savel'ev, Rectification currents in twodimensional artificial channels, Phys. Rev. E 80, 011120 (2009).

[16] P. K. Ghosh, P. Hänggi, F. Marchesoni, and F. Nori, Giant negative mobility of Janus particles in a corrugated channel, Phys. Rev. E 89, 062115 (2014).

[17] E. M. Conwell, Negative differential conductivity, Phys. Today 23, 35 (1970).

[18] F. Nava, C. Canali, F. Catellani, G. Gavioli, and G. Ottaviani, Electron drift velocity in high-purity Ge between 8 and $240 \mathrm{~K}$, J. Phys. C 9, 1865 (1976).

[19] D. I. Aladashvili, Z. A. Adamiya, K. G. Lavdovskii, E. I. Levin, and B. I. Shklovskii, Negative differential resistance in the hopping-conductivity region in silicon, Pis'ma Zh. Eksp. Teor. Fiz. 47390 (1988) [JETP Lett. 47, 466 (1988)].

[20] R. Eichhorn, J. Regtmeier, D. Anselmetti, and P. Reimann, Negative mobility and sorting of colloidal particles, Soft Matter. 6, 1858 (2010).

[21] N. Van Lien and Shklovskii, Hopping conduction in strong electric fields and directed percolation, Solid State Commun. 38, 99 (1981).

[22] H. Böttger and V. V. Bryksin, Hopping conductivity in ordered and disordered systems(III), Phys. Status Solidi B 113, 9 (1982).

[23] E. Lippiello, F. Corberi, and M. Zannetti, Off-equilibrium generalization of the fluctuation dissipation theorem for Ising spins and measurement of the linear response function, Phys. Rev. E 71, 036104 (2005).
[24] M. Baiesi, C. Maes, and B. Wynants, Fluctuations and Response of Nonequilibrium States, Phys. Rev. Lett. 103, 010602 (2009).

[25] M. Baiesi, C. Maes, and B. Wynants, The modified SutherlandEinstein relation for diffusive non-equilibria, Proc. R. Soc. London A 467, 2792 (2011).

[26] S. Mickel, V. Arena, and W. Bauer, Physical properties and gel electrophoresis behavior of R12-derived plasmid DNAs, Nucl. Acids Res. 4, 1465 (1977).

[27] S. D. Levene and B. H. Zimm, Separations of open-circular DNA using pulsed-field electrophoresis, Proc. Natl. Acad. Sci. USA 84, 4054 (1987).

[28] S. Katz, J. L. Lebowitz, and H. Spohn, Phase transitions in stationary nonequilibrium states of model lattice systems, Phys. Rev. B 28, 1655 (1983).

[29] D. T. Gillespie, Exact stochastic simulation of coupled chemical reactions, J. Phys. Chem. 81, 2340 (1977).

[30] S. R. McGuffee and A. H. Elcock, Diffusion, crowding \& protein stability in a dynamic molecular model of the bacterial cytoplasm, PLoS Comput. Biol. 6, e1000694 (2010).

[31] F. Höfling and T. Franosch, Anomalous transport in the crowded world of biological cells, Rep. Prog. Phys. 76, 046602 (2013).

[32] I. Goychuk, V. O. Kharchenko, and R. Metzler, How molecular motors work in the crowded environment of living cells: Coexistence and efficiency of normal and anomalous transport, PLoS ONE 9, e91700 (2014). 